\title{
La Bibliografía Latinoamericana de la UNAM, a propósito de los 350 años de la primera revista científica
}

The Latin-American Bibliography of the UNAM, apropos of the 350 years of the first scientific journal

Felipe Rafael REYNA ESPINOSA

Dirección General de Bibliotecas, Circuito de la Investigación Científica, Ciudad Universitaria, C.P. 04510, México, D.F., México. frre@unam.mx

\begin{abstract}
Resumen
A propósito de los 350 años del nacimiento de la primera revista académica, se hace un recuento de la importancia de la revista como instrumento de comunicación científica y de los esfuerzos de América Latina por compilar, resguardar, organizar y difundir sus revistas académicas y la información científica que contienen. Se resalta la contribución que realiza la Universidad Nacional Autónoma de México (UNAM) a través de su Bibliografía Latinoamericana. Finalmente, se hace una prospectiva de la evolución de las revistas académicas y de los sistemas de información en la región.
\end{abstract}

Palabras clave: Revistas académicas. Bases de datos. Servicios de información. Bibliografía Latinoamericana. Base de datos Clase. Universidad Nacional Autónoma de México (UNAM). América Latina. México.

\section{Introducción}

Con motivo del 350 aniversario de la revista Philosophical Transactions, la Royal Society ha organizado una serie de conferencias sobre el futuro de la revista y la publicación científica en el mundo. En estas reuniones de expertos se ha debatido sobre el papel que deben desarrollar las revistas, sus procesos editoriales, sus modelos de desarrollo y sobre la necesidad de mejorar el modelo de comunicación científica (Gielas, 2015). Consecuentemente, es éste un buen momento para abordar la prospectiva de la revista científica latinoamericana a partir de la experiencia en el desarrollo del conjunto de bases de datos que constituyen la Bibliografía Latinoamericana producida por la Universidad Nacional Autónoma de México (UNAM).

Dejando de lado la polémica de cuál fue verdaderamente la primera revista científica, ya sea la Journal des Sçavants por su fecha de aparición (enero de 1665), o bien, la Philosophical Transactions (marzo de 1665) por su contenido, el hecho importante es que con la revista nace una institución científica y cultural que ha impactado enormemente la generación, validación y difu-

\begin{abstract}
Regarding the 350th anniversary of the birth of the first scholarly journal, the article presents an account of the importance of the journal as an instrument for the communication of science, as well as the Latin American efforts to compile, preserve, organize and disseminate their scholarly journals and the scientific information they contain. The contribution to this goal made by the National Autonomous University of Mexico (UNAM) through the production of its Latin American Bibliography is highlighted. Finally, a prospective of the evolution of scholarly journals and information systems in the region is done.
\end{abstract}

Keywords: Scholarly journals. Databases. Information systems. Latin American Bibliography. Clase database. National Autonomous University of Mexico (UNAM). Latin America. Mexico.

sión de la ciencia en el mundo. Quizá el impacto más evidente es que la revista sentó las bases de la ciencia moderna en el avance de la comunicación científica, a la que dio un medio y una organización más estables que las comunicaciones informales, y a menudo personales, que le antecedieron. En el transcurso de su ya larga vida, los procesos editoriales y los medios y soportes de difusión evolucionaron, pero su esencia se mantiene hasta nuestros días. Cabe mencionar que ambas revistas continúan vigentes, aunque el Journal de Savants con énfasis en temas literarios.

La historia de la evolución de la revista científica desde 1665 hasta nuestros días ha sido ampliamente documentada. Baste decir que en el éxito de las revistas académicas y las bases de datos que las organizan, preservan y difunden, han intervenido instituciones muy importantes como las asociaciones científicas y profesionales y posteriormente las universidades, los organismos de investigación y varias organizaciones internacionales (Cetto y Alonso, 1998; Alonso y Russell, 2012). 
En 1772, en la América novohispana, posteriormente transformada en América Latina, término geográfico que incluye al Caribe, nació El Mercurio Volante, la primera revista médica de América, en el territorio hoy conocido como México. Sin embargo, no fue sino hasta bien entrado el siglo veinte cuando surgieron las primeras reuniones de expertos con el objetivo de conocer mejor las revistas científicas y académicas editadas en la región; prueba de ello fueron las reuniones de científicos, editores y bibliotecarios celebradas en Río Piedras, Puerto Rico en 1964, así como los talleres de publicaciones científicas de Guadalajara, México, celebradas en 1994 y 1997 (Cetto y Alonso, 1998).

Particularmente en México, han sido clave en el fomento, organización, preservación y difusión de las revistas académicas: la UNESCO y su Centro de Información y Documentación (Pérez, 1982); el Consejo Nacional de Ciencia y Tecnología (CONACyT) con su política científica y de fomento (Gama y Vanderkast, 2006); la Universidad Autónoma del Estado de México (UAEM) por la creación del sistema de información Redalyc (Red de Revistas Científicas de América Latina y el Caribe, España y Portugal), y la UNAM a través del conjunto de bases de datos conocido como Bibliografía Latinoamericana.

A pesar de los esfuerzos que se han hecho por acabar con el "ostracismo" de la revista latinoamericana, algunos problemas persisten, como su carácter local (muchas de ellas son publicadas únicamente en español o portugués), recursos humanos y financieros escasos, frecuentes retrasos en su periodicidad y continuidad, así como una muy escasa inclusión en los principales servicios de información internacionales, dando lugar a una situación de marginalidad (Arguello y Márquez, 2008; Alonso y Russell, 2012).

Por estas razones, instituciones como la UNAM han impulsado la creación de sistemas de información sobre sus propias revistas, considerando aspectos sociológicos y humanísticos que se adhieren a los principios de la libre circulación de las ideas y de los resultados del trabajo científico en beneficio de la sociedad. Existe una gran responsabilidad "para defender el derecho a la información, a su fácil acceso y, en consecuencia, a hacer realidad el derecho a la educación y al conocimiento" (Morales, 2013). Estas acciones permiten construir un patrimonio intelectual propio, pues lo contrario implicaría perecer al corto plazo y hacer invisible el trabajo científico regional (Mendoza y Paravic, 2006).

Es así que los sistemas de información latinoamericanos nacieron de universidades y orga- nismos públicos, bajo una filosofía de acceso libre y gratuito, sin duda, un antecedente importante del acceso abierto que sería formalizado en 2002 y 2003. En cambio, en los países hoy considerados como desarrollados, el acceso a los contenidos de revistas científicas quedó en manos del sector privado, sustentado en la obtención de utilidades económicas, lo que marca una gran diferencia con el modelo de desarrollo latinoamericano.

Los sistemas de información y los índices bibliográficos son actualmente una fuente indispensable no sólo para la actualización científica, sino para el establecimiento de rankings del desempeño de grupos de investigación, de instituciones, áreas temáticas, países $y$, desde luego, de las propias revistas (Spinak y Packer, 2015).

\section{La Bibliografía Latinoamericana de la UNAM}

La Bibliografía Latinoamericana (BL) nace en el Centro de Información Científica y Humanística $(\mathrm{CICH})$ de la UNAM y su producción está hoy a cargo de la Dirección General de Bibliotecas (DGB). La BL se compone actualmente de cinco bases de datos:

- Clase - Citas Latinoamericanas en Ciencias Sociales y Humanidades (creada en 1975)

- Periódica - Índice de Revistas Latinoamericanas en Ciencias (1978)

- Latindex - Sistema Regional de Información en Línea para Revistas Científicas de América Latina, el Caribe España y Portugal (1997)

- SciELO México - Scientific Electronic Library Online) (2005)

- Biblat - Portal bibliométrico de la Bibliografía Latinoamericana (2009)

Clase, la primera base de datos de la BL, está especializada en revistas de ciencias sociales y humanidades, lo cual no resulta extraño si se considera que América Latina publica más del $50 \%$ de sus revistas en esas áreas (Alonso y Reyna, 2012). El 40 aniversario de Clase, cumplido en 2015, marca el acontecimiento de una labor constante y creciente de la Bibliografía Latinoamericana, que ha originado la creación de otros productos y, a la vez, de lazos de cooperación con otras instituciones nacionales e internacionales que han favorecido el desarrollo y la calidad de las revistas de la región.

Para las bases de datos que conforman la $\mathrm{Bi}$ bliografía Latinoamericana, la publicación elec- 
trónica y el avance en las tecnologías de información y comunicación han marcado cambios fundamentales que han incrementado su potencial para organizar y difundir información, dejando atrás medios y tecnologías que dejaron de ser efectivos.

Las revistas también han sentido el efecto de esta evolución, ya que paulatinamente fueron abandonando los medios mecánicos de producción e integrando los medios electrónicos disponibles. De esta manera, el ciclo de la mayoría de las revistas empieza y termina de manera electrónica, desde la redacción de los artículos, las comunicaciones y procesos editoriales, hasta llegar al diseño editorial en una computadora para dar al artículo su presentación final.

A partir de aquí, depende únicamente de la política editorial de cada revista si el artículo es difundido en formato electrónico o si aún debe esperar para ser enviado a la imprenta para recorrer el lento camino de la revista impresa.

Los avances tecnológicos permiten hoy que comunidades científicas diversas y distantes entren en contacto, colaboren y contribuyan horizontalmente, de manera que revistas que eran consideradas nacionales, comiencen paulatinamente a convertirse en internacionales por causa de su difusión mundial a través de Internet, por las contribuciones extranjeras que reciben y por la posibilidad de que especialistas de otras latitudes colaboren como dictaminadores o miembros de su comités.

Existe una sinergia entre los productos que componen la Bibliografía Latinoamericana. Por ejemplo, el portal Biblat conjunta indicadores bibliométricos a partir de la información contenida en Clase, Periódica y SciELO México. Este portal ha incrementado su visibilidad de acuerdo con las cuantificaciones de Google Analytics que muestran que de enero de 2014 a mayo de 2015 obtuvo más de un millón de consultas, $49 \%$ de México y $51 \%$ del resto del mundo, principalmente de países de Iberoamérica. Asimismo, en 2014, Biblat ocupó el lugar 104 en el ranking de portales de acceso abierto elaborado por Webometrics, precedido en México sólo por Redalyc y SciELO México.

De acuerdo con información interna del Departamento de Bibliografía Latinoamericana de la DGB, la difusión de las bases de datos en Biblat ha tenido como efecto incrementar sustancialmente las solicitudes de documentación de artículos de Clase y Periódica que no están en texto completo. Estas solicitudes provienen de 59 países, principalmente de México, Brasil, Chile y Perú, y de lugares tan lejanos como Arabia Saudita, Gabón y Senegal.

\subsection{Logros}

Cuarenta años de pervivencia no es algo frecuente en la vida de un emprendimiento y menos aún en el ámbito latinoamericano. En este lapso la BL ha conjuntado un importante acervo de datos sobre las revistas académicas latinoamericanas contribuyendo de esta manera a la infodiversidad, concepto que implica reconocer las diferencias de contenidos y de estilos en la información que existe en el mundo, la cual debe reflejarse en las bibliotecas, en las bases de datos o en las redes de información (Morales, 2006).

Otra aportación es la difusión de la ciencia y la cultura en castellano, a la existencia de conjuntos de información en esta lengua, permitiendo que gane espacios en foros internacionales. En este sentido, los participantes en el Seminario Pensar en Español, celebrado en la UNAM, manifestaron que (Ayala et. al., 2009)

[...] es fundamental poner en Internet la riqueza del conocimiento generado en el mundo hispanoparlante" y "reforzar la visibilidad de las publicaciones locales mediante hemerotecas virtuales que contengan revistas científicas y divulgativas en español, y realizar un esfuerzo para ordenar e indizar la producción latinoamericana.

Quizás el logro más importante de la Bibliografía Latinoamericana, se encuentra en la actitud proactiva, innovadora y pionera de la UNAM en la sistematización de la información científica iberoamericana (Torres y García, 2008).

\subsection{Retos}

Entre los más relevantes se encuentran ampliar la cobertura del número de revistas analizadas y abatir el rezago en la información; para ello debe aumentarse el número de analistas responsables del ingreso de datos.

Dado que una buena parte de la información se encuentra en revistas impresas que no están disponibles en soporte electrónico, otro reto es convertir a soporte digital los acervos impresos de la colección retrospectiva de la Hemeroteca Latinoamericana donde se resguardan los ejemplares impresos que se analizan en Clase y Periódica.

Los cambios tecnológicos obligan también a migrar a manejadores de bases de datos que permitan ofrecer a los usuarios más y mejores funcionalidades.

Otro aspecto importante es obtener el apoyo institucional necesario para continuar la pervivencia de la Bibliografía Latinoamericana. 


\section{Prospectiva de las revistas latinoamericanas}

A partir de la experiencia en la producción de la Bibliografía Latinoamericana, se pueden observar algunas tendencias en la edición de las revistas científicas editadas en la región:

Soporte electrónico. Las tecnologías de la información y comunicación son ya utilizadas en todo el proceso editorial de una revista y los nuevos desarrollos en el campo de las revistas científicas están basados principalmente en el formato electrónico. Se empieza a detectar una tendencia a publicar más revistas en soporte digital. Por ejemplo, en México a partir del año 2008 se crean más revistas electrónicas que impresas (Alonso y Reyna, 2015).

No obstante, en América Latina muchas revistas siguen apostando por publicar en ambos soportes, a pesar del consumo reiterado en tiempo y recursos. Posiblemente esta orientación cambie cuando se hagan más evidentes las inconveniencias que el formato impreso impone a la comunicación científica.

Calidad editorial. El trabajo realizado en las últimas décadas por editores, bases de datos e iniciativas de información regionales como Latindex, Redalyc y SciELO ha aumentado de manera significativa la adopción de criterios de calidad editorial, al grado que algunas revistas han llamado la atención de empresas editoriales como Elsevier y Thomson Reuters para integrarlas a sus servicios. En algunos casos son incluidas como parte de sus colecciones y servicios, o bien editadas directamente por estas empresas. Un ejemplo claro de este proceso lo constituye el acuerdo que dio lugar al SciELO Citation Index, que integra un subconjunto de la colección SciELO, accesible a través de la plataforma de Thomson Reuters.

Hemerotecas virtuales. Cada vez son más las revistas que se integran a estas plataformas, ya que aportan valores añadidos a las revistas como estabilidad, consistencia, normalización, metodología, indicadores bibliométricos, además de otras funcionalidades. La inclusión de una revista en estos servicios aumenta su calidad, difusión, prestigio y alcance.

Acceso abierto. Se ha implantado sólidamente en la región aunque con algunas resistencias. En México se está impulsando una política nacional que favorece el acceso libre, así como la transformación de las revistas al soporte digital. En 2014 se aprobó el decreto que promueve el acceso abierto, la creación de repositorios y el depósito de obras de carácter académico, científico o artístico que hayan sido financiadas con fondos públicos, aunque este depósito se establece de manera opcional y no obligatoria (Diario Oficial, 2014).

Internacionalización. La adecuada utilización de las tecnologías de información y comunicación ha permitido a las revistas romper su aislamiento, ampliando su alcance hacia otras regiones del mundo. La adopción de criterios de calidad les ha permitido integrar cuerpos editoriales $y$ revisores multinacionales; también han redoblado sus esfuerzos por conseguir contribuciones de autores adscritos a instituciones extranjeras.

Sustentabilidad. Recientemente se ha detectado una "deserción" de revistas académicas latinoamericanas desde plataformas de acceso abierto como SciELO, hacia plataformas de editores comerciales. En algunos casos las revistas mantienen el acceso abierto a su información, pero otras han optado por las suscripciones pagadas, posiblemente como una estrategia de supervivencia. Esta situación sugiere que aún falta consolidar un modelo de desarrollo que concilie los intereses académicos y de sustentabilidad de las revistas, con los intereses económicos de los editores comerciales.

De igual manera, son ya varias las revistas latinoamericanas cuya edición está ahora a cargo de empresas editoriales extranjeras como una estrategia para incrementar su visibilidad internacional. Sin embargo, se ha dado lugar a una especie de "desnacionalización", ya que en ocasiones el proceso ha implicado el cambio de títulos al inglés y la asignación de un ISSN extranjero, lo que ha ocasionado que salgan de servicios de información de orientación nacional o regional como SciELO, aunque el órgano académico responsable de esas revistas siga siendo una institución latinoamericana.

Métrica. Existe un marcado interés por ofrecer métricas a nivel de artículo en las bases de datos y hemerotecas digitales. A nivel latinoamericano SciELO, Biblat y Redalyc han trabajado en este sentido, ofreciendo cuantificaciones que hasta hace algunos años no existían para las revistas latinoamericanas.

Usuarios. Los usuarios (lectores y autores) de las revistas académicas poseen ahora una cultura informativa más amplia y sus preferencias se orientan a aquellas fuentes de información que ofrecen el texto completo de los materiales de su interés, que estén incluidas en servicios de información de prestigio internacional y que son reconocidas por los sistemas evaluadores en sus países. 


\section{Reflexiones y conclusiones}

La revista académica ha experimentado avances tecnológicos y editoriales muy importantes en los últimos veinte años y se le ha prestado atención como nunca antes en su larga existencia.

En América Latina, las reuniones de expertos han sido cruciales para el avance de la revista académica latinoamericana. De estas reuniones se han desprendido diagnósticos y recomendaciones que han incidido en la organización de los flujos de información científica y en el desarrollo de productos de información como los que ofrece la Bibliografía Latinoamericana. En esta labor las universidades e instituciones de investigación académica han sido los principales impulsores de las revistas.

Aunque el devenir de la revista académica ha generado cambios inéditos en su producción, el apoyo institucional, la profesionalización del trabajo editorial y la calidad se mantienen como elementos siempre presentes en los proyectos editoriales exitosos.

Desde esta perspectiva, las tendencias más importantes en el campo de las revistas académicas se dirigen al apego a criterios de calidad editorial y normas, el soporte electrónico, la adhesión a hemerotecas virtuales y la adopción de políticas de acceso abierto, del cual América Latina es precursora. Estamos ante una etapa que llama a compartir esfuerzos y a eliminar obstáculos para la libre difusión de nuestras revistas y sus contenidos.

Sin duda, la revista académica llega al siglo XXI con una sólida posición como el principal medio para la validación y divulgación de los avances y descubrimientos científicos y también como el medio ideal para la rápida difusión de las ideas. Estas funciones de las revistas se han visto reforzadas con la aparición de la publicación electrónica e Internet. Estas nuevas herramientas de la comunicación científica permiten no solamente la difusión amplia y expedita de los contenidos de las revistas, sino también el aprovechamiento pleno de las potencialidades de colaboración internacional.

Posiblemente ahora estemos más cerca de aquella visión de Eugene Garfield expresada en su visita a la UNAM en 1976, cuando vislumbraba que la universidad del futuro tendría el cometido de producir y consumir información y de ejercer su responsabilidad como educadora de la humanidad, proveyendo todos los medios imaginables para permitir a estudiantes, maestros, investigadores y administradores "obtener información rápida y eficazmente de todas par- tes del mundo, integrando simultáneamente en el sistema sus propias ideas y pensamientos" (Garfield, 1983).

\section{Referencias}

Alonso Gamboa, José Octavio; Reyna Espinosa, Felipe Rafael (2012). La revista iberoamericana en Latindex, una visión de 15 años. // Biblioteca universitaria. 15:2 (julio-diciembre 2012) 123-138. http://132.248.9.34/he vila/Bibliotecauniversitaria/2012/vol15/no2/1.pdf (201505-07).

Alonso Gamboa, José Octavio; Reyna Espinosa, Felipe Rafael (2015). Revistas académicas mexicanas. Panorama y prospectiva. // Ciencia ergo sum. 22:3 (noviembre 2015 - febrero 2016) 181-191. [En prensa].

Alonso-Gamboa, José Octavio; Russell, Jane M. (2012). Latin American scholarly journal databases: a look back to the way forward. // Aslib Proceedings: New Information Perspectives. 64:1 (2012) 32-45. http:// dx.doi.org/10.1108/00012531211196693 (2015-04-23).

Arguello Mendoza, Maria Guadalupe; Márquez Rangel, Sergio (2008). Visibilidad de las revistas electrónicas latinoamericanas en las bases de datos internacionales. // lbersid. 2 (2008) 85-92. http://www.ibersid.eu/ojs/index. php/ibersid/article/view/2209/1970 (2015-04-29).

Ayala, Gustavo; Juárez, Aline; López, Patricia; Olvera Leticia; Romero, Laura (2009). Necesario revitalizar el idioma español. // Gaceta UNAM. 4192 (8 de octubre, 2009) 8-9. http://www.acervo.gaceta.unam.mx/index.php /gum00/article/download/18305/18295 (2015-05-11).

Biblat - (Portal Bibliografía Latinoamericana). http://biblat. unam.mx/es/ (6 de junio de 2015).

Cetto, Ana María; Alonso, Octavio (1998). Scientific periodicals in Latin America and the Caribbean: a global perspective. // Interciencia. $23: 2$ (1998) 84-93. http://www. interciencia.org/v23_02/cetto.pdf (2015-04-06).

Clase - Citas Latinoamericanas en Ciencias Sociales $y$ Humanidades. http://clase.unam.mx/ (2015-06-06).

Diario Oficial de la Federación de México (2014). Decreto por el que se reforman y adicionan diversas disposiciones de la Ley de Ciencia y Tecnología, de la Ley General de Educación y de la Ley Orgánica del Consejo Nacional de Ciencia y Tecnología. (20 mayo de 2014). http://www.dof.gob.mx/nota detalle.php?codigo $=534550$ 3\&fecha=20/05/2014 (2015-05-29).

Gama, Miguel; Vanderkast, Egbert (2006). Tópicos de políticas de información en el entorno científico y técnico: México 1989 -1994. // Ciencia da Informacao. 35:3 (setembro-dezembro 2006) 75-88. http://www.scielo.br/pdf/ ci/v35n3/v35n3a08.pdf (2015-04-08).

Garfield, Eugene (1983). Las universidades como productoras y consumidoras de información. // Inforum. 2 (1983) 15 p. http://132.248.9.34/hevila/Inforum/1983/no2/2.pdf (2015-06-30).

Gielas, Anna. After 350 years of academic journals it's time to shake things up. // The Guardian. (4 de abril de 2015). http://www.theguardian.com/science/political-science/20 15/apr/04/after-350-years-of-academic-journals-its-timeto-shake-things-up (2015-04-10).

Latindex - Sistema Regional de Información en Línea para Revistas Científicas de América Latina, el Caribe España y Portugal. http://www.latindex.unam.mx/ (2015-0606)

Mendoza, Sara; Paravic, Tatiana (2006). Origen, clasificación y desafíos de las revistas científicas. // Investigación y postgrado. 21:1 (2006) 49-75 http://dialnet.uni rioja.es/descarga/articulo/2309841.pdf (2015-05-12). 
Morales Campos, Estela (2013). Acceso a la educación y al conocimiento a partir del derecho a la información, p. 115-133. En: Regulaciones que impactan la infodiversidad y el acceso abierto a la información en la sociedad global y multicultural. México: UNAM, Instituto de Investigaciones Bibliotecológicas y de la Información, 2013. ISBN: 978-607-02-4885-6. http://132.248.9.34/cuib/1655 455.pdf (2015-06-02).

Morales Campos, Estela (coord) (2006). Infodiversidad y cibercultura: globalización e información en América Latina. Buenos Aires: Alfagrama, 2006, 172 p. ISBN 9871305-14-1.

Pérez Vitoria, Augusto (1982). La información en el marco de la cooperación científica internacional. // Inforum. 1 (1982) 1-31. http://132.248.9.34/hevila/Inforum/1982/no 1/1.pdf (2015-04-23) .

Periódica - Índice de Revistas Latinoamericanas en Ciencias. http://periodica.unam.mx/ (2015-06-02).

Redalyc - (Red de Revistas Científicas de América Latina y el Caribe, España y Portugal). http://www.redalyc.org/ (2015-07-02)
SciELO México - Scientific Electronic Library Online). http://www.scielo.org.mx (2015-06-02).

Spinak, Ernesto; Packer, Abel L. (2015). 350 años de publicación científica: desde el "Journal des Sçavans" y el "Philosophical Transactions" hasta SciELO. // SciELO en Perspectiva. 5 de marzo, 2015. http://blog.scielo.org/es/ 2015/03/05/350-anos-de-publicacion-cientifica-desde-eljournal-des-scavans-y-el-philosophical-transactions-hast a-scielo/ (2015-04-09).

Torres Verdugo, María Ángela; García Colorado, Carmen (2008). La Universidad Nacional Autónoma de México: pionera en la sistematización de la información científica iberoamericana. // Ibersid. (2008) 117-122. http://ibersid. eu/ojs/index.php/ibersid/article/download/2215/1976 (2015-04-24).

Enviado: 2015-07-28. Segunda versión: 2015-06-15 Aceptado: 2015-06-19. 